\title{
A longitudinal study of neck and upper limb musculoskeletal disorders and alternative measures of vibration exposure
}

\author{
Massimo Bovenzi 1,* \\ Phone +39-040-3992313 Phone +39-040-632797 Email bovenzi@units.it \\ Andrea Prodi 1 \\ Marcella Mauro 1 \\ 1 Clinical Unit of Occupational Medicine, Department of Medical Sciences, University of Trieste, Centro Tumori, Via della Pietà \\ 19, 34129 Trieste, Italy
}

\begin{abstract}
Objective

To investigate the exposure-response relationships between alternative frequency weightings of hand-transmitted vibration (HTV) and neck and upper limb musculoskeletal disorders (MSDs) in a cohort of HTV workers.
\end{abstract}

\section{AQ1}

\section{Methods}

In a three-year longitudinal study, the occurrence of neck and upper limb MSDs was investigated in 249 HTV workers and 138 control men. In the HTV workers, MSDs were related to measures of daily vibration exposure expressed in terms of 8-h energyequivalent frequency-weighted acceleration magnitude $[A(8)]$. To calculate $A(8)$, the acceleration magnitudes of vibration were weighted by means of four alternative frequency weightings of HTV. The associations between MSDs, individual characteristics, physical work load other than vibration, and psychological strain were also investigated. 


\section{Results}

The occurrence of upper limb MSDs was greater in the HTV workers than in the controls. After adjustment for potential confounders, the occurrence of elbow/forearm and wrist/hand MSDs increased with the increase in vibration exposure. A measure of model selection did not reveal any substantial difference in the performance of the alternative frequency weightings of HTV for the prediction of neck and upper limb MSDs. In the study population, age, hard physical work load, and poor psychological well-being were associated with both neck and upper limb MSDs.

\section{Conclusions}

In this study, there was evidence for significant exposure-response relationships between HTV exposure and MSDs in the distal sites of the upper limbs. There were no differences in the prediction of neck and upper limb MSDs between measures of daily vibration exposure calculated with alternative frequency weightings of acceleration magnitude.

\section{Keywords}

Frequency weightings

Hand-transmitted vibration

Neck and upper limb musculoskeletal disorders

Physical work load

Prospective cohort study

Psychological strain

\section{Introduction}

The role of hand-transmitted vibration (HTV) in the etiopathogenesis of neck and upper limb musculoskeletal disorders (MSDs) is still debated. This may be due, at least partially, to the fact that there are no universally accepted definitions and criteria for the 
diagnosis of work-related MSDs, although consensus documents and reviews have proposed minimum diagnostic criteria to differentiate specific from non-specific MSDs (Harrington et al. 1998; Huisstede et al. 2007).

Since work with vibratory tools involves not only exposure to HTV but also to additional physical loads, such as repetitive or forceful movements and/or awkward postures, it is hard to distinguish the relative contributions of these risk factors to the occurrence of MSDs. In some European countries (e.g. France, Germany, and Italy), tendon, muscle, bone, and joint disorders in HTV workers are recognised as occupational diseases and, under certain conditions regarding intensity and duration of exposure, the affected workers are compensated. In the Recommendation 2003/670 of the European Commission for the adoption of a European schedule of occupational diseases (2003), the "osteoarticular diseases of the hands and wrists caused by mechanical vibration” (No. 505.01 of the list in Annex I) are recognised to be linked directly to the occupation, and as such liable for compensation and subject to preventive measures.

The term "hand-arm vibration syndrome" (HAVS) is used to denote collectively the vascular, neurological, and musculoskeletal disorders associated with occupational exposure to HTV (CEN 1996). In an annex to International Standard ISO 5349-1 (2001), an exposure-response relationship is proposed for the prediction of vibration-induced disorders, notably with reference to the vascular component of the HAVS named vibration-induced white finger. In the ISO standard, daily exposure to HTV is expressed in terms of 8-h energy-equivalent frequency-weighted root-mean-square (r.m.s.) acceleration magnitude [A(8) in $\mathrm{m} \mathrm{s} \mathrm{s}^{-2}$ r.m.s.]. Biodynamic investigations have shown that the response of the hand-arm system to vibration is frequency-dependent. To account for this feature, ISO 5349-1 recommends to weigh the vibration by means of a frequency weighting function (called $W_{\mathrm{h}}$ ) which assumes that the adverse health effects of HTV are approximately proportional to the frequency of vibration between 6.3 and $16 \mathrm{~Hz}$, and inversely related to the frequency in the range 16-1250 Hz. Since the findings of biodynamic, physiological, and epidemiological investigations have questioned the suitability of the ISO frequency weighting to assess all disorders caused by HTV, recently a set of candidate frequency weightings has been proposed for the measurement and evaluation of vibration exposure at the workplace. A common characteristic of the candidate frequency weightings is to give more weight to intermediateand high-frequency vibrations compared to the ISO weighting function $W_{\mathrm{h}}$ (Pitts et al. 2012).

\section{AQ3}

The aims of this longitudinal study were (1) to investigate the prevalence and incidence of neck and upper limb MSDs in a cohort of HTV workers recruited in a four-year research project supported by the EU Commission (VIBRISKS 2007); (2) to evaluate the relative performance of the ISO frequency weighting and three candidate frequency weightings of HTV to estimate the occurrence 


\section{Subjects and methods}

\section{Interview and medical investigations}

The study design and the characteristics of the study population have been described in detail in previous papers (Bovenzi 2010; Bovenzi et al. 2011). Briefly, 249 HTV workers (215 forestry operators and 34 stone workers) and 138 male controls employed at the same companies and unexposed to HTV (maintenance operators, inspectors, supervisors) participated in a cross-sectional survey and three annual follow-up investigations in autumn-winter 2003-2007. Of the HTV workers, 177 participated in three follow-ups, 36 in two follow-ups, and 36 in one follow-up survey. Of the controls, 99 participated in three follow-ups, 19 in two follow-ups, and 20 in one follow-up survey. Reasons for dropping out of the follow-ups were change in residence, retirement, and refusal to participate in the follow-up (Bovenzi 2010).

\section{AQ4}

All HTV workers continued to operate vibratory tools during the follow-up.

Informed consent was given by all subjects, and the study was approved by the ethical committee of the local health authorities.

Personal, work, and health histories were investigated by means of a structured questionnaire developed within the EU research project VIBRISKS (2007). The medical interview and a complete physical examination focused on the vascular, neurological, and musculoskeletal systems of the neck and upper limbs were carried out by the same certified occupational physicians at the initial and follow-up surveys.

The medical investigation of MSDs in the neck and/or upper limbs sought for (1) symptoms of pain, stiffness, weakness, and/or swelling in the neck/upper limb anatomical areas (shown in a body map) lasting 1 day or longer in the last 12 months; (2) abnormal signs at the physical examination such as local pain and tenderness over tendon insertion, restricted or painful active and passive movements of the neck and/or upper limbs, pain during resisted extension/flexion, palpable hardenings and tender spots in tendons or muscles, decreased strength in gripping, Finkelstein's test for de Quervain's disease, Spurling manoeuvre for cervical radiculopathy. 
A subject who reported one or more symptoms and had one or more abnormal findings on examination at the same body location (neck, shoulder/upper arm, elbow/forearm, wrist/hand) was defined a case of MSD (Punnett et al. 2004). Since symptoms and signs were not combined into definite categories for the clinical diagnosis of specific MSDs, this study focused on non-specific regional MSDs in the neck and upper limbs of the HTV workers at both the cross-sectional and follow-up surveys.

Neck or upper limb traumatic injuries and/or surgery in the past or over the follow-up period were also investigated.

\section{Measurement and evaluation of vibration exposure}

Vibration was measured in three orthogonal directions $(x, y$, and $z)$ on the handles of the vibratory tools used by the forestry operators (brush saws, chain saws) and the stone workers (grinders, polishers, inline hammers) according to the procedure recommended by ISO 5349-1 (2001). Details on vibration measurements are reported elsewhere (Bovenzi 2010). Vibration signals were weighted by means of the ISO frequency weighting and three candidate frequency weightings shown in Fig. 1:

\section{Fig. 1}

Comparison of frequency weighting functions for hand-transmitted vibration. $W_{\mathrm{h}}$ : frequency weighting as defined in ISO 5349-1 (2001); $W_{\mathrm{h}-\mathrm{bl}}$ : the band-limiting component of $W_{\mathrm{h}}$ (ISO 5349-1: 2001); $W_{\mathrm{hf}}$ : a frequency weighting based on finger vibration power absorption (Dong et al. 2005); $W_{\mathrm{hT}}$ : a frequency weighting based on a Japanese study of VWF prevalence (Tominaga 2005)

1. $W_{\mathrm{h}}$ is the frequency weighting specified in ISO 5349-1 (2001);

2. $W_{\mathrm{h}-\mathrm{bl}}$ is the band-limiting component of $W_{\mathrm{h}}(2001)$;

3. $W_{\text {hf }}$ is a frequency weighting based on the biodynamic studies of the vibration power absorption in the hand-arm system (Dong et al. 2005);

4. $W_{\mathrm{hT}}$ is a frequency weighting based on a prevalence study of vibration-induced injuries in Japanese workers (Tominaga 2005). 
Compared to the ISO frequency weighting $\left(W_{\mathrm{h}}\right)$, the additional candidates for frequency weighting of HTV greatly increase the contribution of intermediate- and high-frequency vibration to the magnitude of frequency-weighted r.m.s. acceleration.

The vibration total value of the r.m.s. acceleration frequency-weighted according to $W_{\mathrm{h}}, W_{\mathrm{h}-\mathrm{bl}}, W_{\mathrm{hf}}$, or $W_{\mathrm{hT}}\left(a_{\mathrm{hv}}\left(W_{\mathrm{hi}}\right)\right)$ for the $x$-, $y$-, and z-axes was calculated according to ISO 5349-1 (2001):

$$
a_{\mathrm{hv}}\left(W_{\mathrm{hi}}\right)=\sqrt{a_{\mathrm{hx}\left(W_{\mathrm{hi}}\right)}^{2}+a_{\mathrm{hy}\left(W_{\mathrm{hi}}\right)}^{2}+a_{\mathrm{hz}\left(W_{\mathrm{hi}}\right)}^{2}}\left(\mathrm{~m} \mathrm{~s}^{-2} \text { r.m. s. }\right)
$$

The results of vibration measurements on the tools $\left(a_{\mathrm{hv}}\left(W_{\mathrm{hi}}\right)\right)$ are reported in a previous study (Bovenzi et al. 2011).

Questionnaire data, interviews of employees and employers, and company records were used to estimate daily (hours) and lifetime (years) duration of tool usage.

Daily vibration exposure was evaluated according to the International Standard ISO 5349-1 (2001) and the European Directive on mechanical vibration (2002), and expressed in terms of 8-h energy-equivalent frequency-weighted r.m.s. acceleration magnitude $\left(A(8) W_{\mathrm{hi}}\right)$ :

$$
A(8) W_{\mathrm{hi}}=\sqrt{\frac{1}{T_{0}} \sum_{i=1}^{n}\left(a_{\mathrm{hvi}}\left(W_{\mathrm{hi}}\right)\right)^{2} T_{\mathrm{i}}}\left(\mathrm{m} \mathrm{s}^{-2} \text { r.m.s. }\right)
$$

where $a_{\mathrm{hvi}}\left(W_{\mathrm{hi}}\right)$ is the vibration total value (frequency-weighted with $W_{\mathrm{h}}, W_{\mathrm{h}-\mathrm{bl}}, W_{\mathrm{hf}}$, or $\left.W_{\mathrm{hT}}\right)$ for tool $i$ in $\mathrm{m} \mathrm{s} \mathrm{s}^{-2} \mathrm{r.m.s.}, T_{i}$ is the daily duration of exposure to tool $i$ in hours, and $T_{0}$ is the reference duration of $8 \mathrm{~h}$.

\section{Physical and psychological load factors}

Physical work load on the neck and upper limbs was investigated by means of 19 questions concerning prolonged or recurrent work with the neck bent and/or twisted, lifting weights $\geq 25 \mathrm{~kg}$, repeated or prolonged carrying, pushing or pulling of loads, repetitive or forceful movements with the forearms and hands, work tasks with the arms stretched forwards or outwards unsupported or above shoulder height, uncomfortable hand positions/grips, and heavy demands on precision (VIBRISKS 2007). Physical load in a 
typical working day was graded by rating the frequency of adverse movements and awkward postures on a four-point response scale [“never” (score 0), “1-4 times” (score 1), “5-20 times” (score 2), and “more than 20 times” (score 3)]. Scores of physical demand were added up for each subject, and in the entire sample, the total score was categorised into quartiles which were assumed to correspond to four grades of increasing physical work load: scores 0-12 = no or mild load grade, scores 1325 = moderate load grade, scores 26-35 = medium load grade, and scores 36-57 = hard load grade.

Psychosomatic and psychological strain in the past 7 days was investigated with four questions ("episodes of headache”, "feeling constantly tired”, “feeling low in mood”, and "feeling under stress”) and rated on a three-point scale: “never” (score 0), “some days" (score 1), and "every day” (score 2). Positive responses to these questions were considered expression of psychosomatic/psychological symptoms after excluding the coexistence of systemic diseases (e.g. cardiovascular and neuropsychiatric) potentially associated with psychological distress. Scores of psychological strain were added up for each subject, and in the total sample, the score sum was categorised into tertiles which were assumed to correspond to three grades of increasing psychological strain: score $0-2=$ no or mild strain, score 3-5 = moderate strain, and score 6-8 = high strain.

\section{Data analysis}

The statistical analysis of data was performed with the Stata software, version 13.1 (Stata Corporation ${ }^{\circledR}$, State College, TX, USA, 2013).

Continuous variables were summarised with the median as a measure of central tendency and quartiles as a measure of dispersion. Comparison between unpaired data was carried out by means of nonparametric statistics.

Categorical data were tabulated into $2 \times 2$ or $2 \times k$ contingency tables, and the difference between proportions was tested by means of the $\chi^{2}$ statistic or the Fisher's exact test.

The relations of MSD (binary) outcomes to alternative measures of vibration exposure were assessed by means of the generalised estimating equations (GEE) method in order to account for the within-subject dependency of the observations over time. Odds ratios (ORs) and robust $95 \%$ confidence intervals (95 \% CI), adjusted by potential confounders, were estimated from the GEE models using a logit link function.

The quasi-likelihood information criterion (QIC), a modification of the Akaike's information criterion (AIC), was used to select the 
best working correlation structure in GEE analyses, and to compare the fit of GEE longitudinal models including alternative measures of daily vibration exposure (Cui 2007). In this study, an autoregressive correlation structure was specified for parameter estimation in GEE analyses since the QIC statistic for this structure had smaller values than those obtained with different working correlations (e.g. exchangeable or unstructured structures). The models with the smallest QIC values were also chosen as the bestfitting models for the relation between outcomes and vibration exposure. To aid comparison, a $\Delta \mathrm{QIC}$ was calculated as the difference between the QIC value for a specific exposure model and the model including $A(8)$ calculated with frequency weighting $W_{\mathrm{h}}$ (i.e. the ISO weighting method). By analogy with the strength of evidence rules suggested for the AIC method (Burnham and Anderson 2004), the following guidelines for selecting the best-fitting model were adopted: $\Delta$ QIC $\leq 2$ suggests no difference in the fit between models; $4 \leq \Delta \mathrm{QIC} \leq 7$ tends to give support for the model with the smaller QIC; $\Delta \mathrm{QIC}>10$ means that the model with the smaller QIC provides a substantially better fit to the data.

\section{Results}

At the initial survey, there were no significant differences between the HTV workers and the control men with respect to individual characteristics and job seniority, with the exception for smoking which was more frequent in the HTV workers (Table 1).

\section{Table 1}

Characteristics of the controls and the workers exposed to hand-transmitted vibration (HTV) at the cross-sectional survey

\begin{tabular}{|c|c|c|c|c|}
\hline \multirow{2}{*}{ Characteristics } & \multirow{2}{*}{$\begin{array}{l}\text { Controls } \\
(n=138)\end{array}$} & \multicolumn{3}{|l|}{ HTV workers } \\
\hline & & $\begin{array}{l}\text { Forestry workers } \\
(n=215)\end{array}$ & $\begin{array}{l}\text { Stone workers } \\
(n=34)\end{array}$ & Total $(n=249)$ \\
\hline Age (year) & $38.8(34.1-45.9)$ & $42.8(34.6-48.2)$ & $37.2(30.9-43.2)$ & $42.1(34.0-47.9)$ \\
\hline Body mass index $\left(\mathrm{kg} \mathrm{m}^{-2}\right)$ & $24.5(23.0-27.2)$ & $25.5(23.2-27.4)$ & $25.6(23.5-26.8)$ & $25.5(23.2-27.1)$ \\
\hline Smoking & $29(21.0)$ & $95(44.2)$ & 18 (52.9) & $113(45.4)^{\mathrm{a}}$ \\
\hline Drinking & $104(75.4)$ & $162(75.3)$ & $30(88.2)$ & $192(77.1)$ \\
\hline Job seniority (year) & $15(7-23)$ & $15(6-21)$ & $17(11-23)$ & $15(8-21)$ \\
\hline Duration of daily HTV exposure & - & $114(72-180)$ & $373(335-413)^{b}$ & $126(72-194)$ \\
\hline
\end{tabular}




\begin{tabular}{|l|l|l|l|l|}
\hline (min) & & & & \\
\hline$A(8) W_{\mathrm{h}}\left(\mathrm{m} \mathrm{s}^{-2}\right.$ r.m.s.) & - & $3.5(2.5-4.8)$ & $6.7(5.4-15.8)^{\mathrm{b}}$ & $3.8(2.8-5.4)$ \\
\hline$A(8) W_{\mathrm{h}-\mathrm{bl}}\left(\mathrm{m} \mathrm{s}^{-2}\right.$ r.m.s. $)$ & - & $18.9(13.2-27.9)$ & $94.2(79.6-193)^{\mathrm{b}}$ & $20.6(14.3-34.6)$ \\
\hline$A(8) W_{\mathrm{hf}}\left(\mathrm{m} \mathrm{s}^{-2}\right.$ r.m.s. $)$ & - & $17.9(12.9-27.3)$ & $78.2(66.1-160)^{\mathrm{b}}$ & $19.8(13.7-32.3)$ \\
\hline$A(8) W_{\mathrm{hT}}\left(\mathrm{m} \mathrm{s}^{-2}\right.$ r.m.s. $)$ & - & $16.3(11.8-24.6)$ & $72.6(61.3-148)^{\mathrm{b}}$ & $18.0(12.3-30.0)$ \\
\hline
\end{tabular}

Physical work load

\begin{tabular}{|c|c|c|c|c|}
\hline No/mild (score 0-12) & $114(82.6)$ & 29 (13.5) & $4(11.8)$ & $33(13.2)$ \\
\hline Medium (score 26-35) & $8(5.8)$ & $83(38.6)$ & $12(35.3)$ & $95(38.2)$ \\
\hline Hard (score 36-57) & $1(0.7)$ & $49(22.8)$ & $7(20.6)$ & $56(22.5)^{\mathrm{a}}$ \\
\hline \multicolumn{5}{|c|}{ Psychological strain in the last 7 days } \\
\hline Moderate (score 3-5) & $26(18.8)$ & $78(36.3)$ & $9(26.4)$ & $87(34.9)$ \\
\hline High (score 6-8) & $6(4.4)$ & $14(6.5)$ & $2(5.9)$ & $16(6.4)^{\mathrm{a}}$ \\
\hline
\end{tabular}

See text for the definition of $A(8) W_{\mathrm{h}}, A(8) W_{\mathrm{h}-\mathrm{bl}}, A(8) W_{\mathrm{hf}}$, and $A(8) W_{\mathrm{hT}}$. Data are given as medians (quartiles) or numbers (\%)

$\chi^{2}$ test (HTV workers vs. controls): ${ }^{\text {a }} P<0.001$

Mann-Whitney test (forestry vs. stone workers): ${ }^{\mathrm{b}} \mathrm{P}<0.0001$

At baseline, physical work load and psychological strain were more prevalent in the HTV workers than in the controls $(P<0.001)$, while no differences were observed between the two HTV groups.

Daily vibration exposure in terms of duration and magnitude $\left(A(8) W_{\mathrm{hi}}\right)$ was significantly greater in the stone workers than in the forestry operators $(P<0.0001)$. 


\section{Occurrence of neck and upper limb MSDs}

The prevalence and the cumulative incidence of MSDs in the shoulder/upper arm, elbow/forearm, and wrist/hand were significantly greater in the HTV workers than in the control men (adjusted ORs over the follow-up period 1.72-2.85), (Table 2). There was no difference in the occurrence of neck complaints between the two groups. Compared with the controls, a two- to threefold increase in the risk of elbow/forearm and wrist/hand MSDs was observed in the forestry operators (ORs 2.38-2.79) and the stone workers (ORs 1.94-3.23).

\section{Table 2}

Prevalence at baseline and cumulative incidence of neck and upper limb musculoskeletal disorders in the controls and the workers exposed to hand-transmitted vibration (HTV) by job title

\begin{tabular}{|c|c|c|c|c|}
\hline Musculoskeletal disorders & Controls $(n=138)$ & \multicolumn{3}{|l|}{ HTV workers } \\
\hline Prevalence at baseline & 55 (39.9) & $84(39.1)$ & $16(47.1)$ & $100(40.2)$ \\
\hline Cumulative incidence & $18(21.7)$ & $34(25.9)$ & $4(22.2)$ & $38(25.5)$ \\
\hline \multicolumn{5}{|l|}{ Shoulder/upper arm } \\
\hline Prevalence at baseline & 17 (12.3) & $70(32.6)^{\mathrm{f}, \mathrm{i}}$ & $5(14.7)$ & $75(30.1)^{\mathrm{C}}$ \\
\hline Cumulative incidence & $15(12.4)$ & $22(15.3)$ & $4(13.8)$ & $26(14.9)$ \\
\hline Prevalence at baseline & $8(5.8)$ & $47(21.9)^{\mathrm{f}}$ & $6(17.7)^{g}$ & $53(21.3)^{\mathrm{c}}$ \\
\hline Cumulative incidence & $13(10.0)$ & $33(19.6)^{\mathrm{d}}$ & $4(14.3)$ & $37(18.9)^{\mathrm{a}}$ \\
\hline
\end{tabular}


OR (95 \% CI)

$1.0(-)$

2.38 (1.69-3.34)

$1.94(1.13-3.32)$

$2.31(1.66-3.23)$

Wrist/hand

Prevalence at baseline

Cumulative incidence

OR (95 \% CI)

$10(8.0)$

$1.0(-)$

2.79 (1.95-4.00)
4 (11.8)

$9(30.0)^{\mathrm{h}}$

3.23 (1.93-5.43) $47(18.9)^{b}$

$45(22.3)^{\mathrm{c}}$

2.85 (2.01-4.06)

Odds ratios (OR) and robust $95 \%$ confidence intervals (95 \% CI) were estimated by means of the generalised estimating equations method for repeated measures over time, assuming the controls as the reference category. Prevalence and incidence data are given as numbers $(\%)$

OR adjusted by age at entry, body mass index, smoking and drinking habits, neck/upper limb traumatic injuries or surgery, and followup time

$\chi^{2}$ or Fisher's exact test: HTV workers versus controls: ${ }^{\mathrm{a}} P<0.05$; ${ }^{\mathrm{b}} P<0.01$; ${ }^{\mathrm{c}} P<0.001$; forestry workers versus controls: ${ }^{\mathrm{d}} P<0.05$;

${ }^{\mathrm{e}} P<0.01$; ${ }^{\mathrm{f}} P<0.001$; stone workers versus controls: ${ }^{\mathrm{g}} \mathrm{P}<0.05$; ${ }^{\mathrm{h}} \mathrm{P}<0.001$; stone workers versus forestry workers: ${ }^{\mathrm{i}} P<0.05$

There were no significant differences between the controls and the HTV workers for neck or upper limb traumatic injuries (20.3 vs. $17.3 \%, P=0.46$ ), and neck or upper limb surgery (5.8 vs. $3.2 \%, P=0.22$ ).

\section{Relationships of neck and upper limb MSDs to alternative measures of daily vibration exposure}

After adjustment for potential confounders, all alternative measures of daily vibration exposure $\left(A(8) W_{\text {hi }}\right)$, expressed as categorical variables, were significantly associated with the occurrence of MSDs in the elbow/forearm and wrist/hand (Table 3). The associations were weaker for shoulder/upper arm disorders and nil for neck disorders.

Table 3

Relations of neck and upper limb musculoskeletal disorders to alternative measures of daily vibration exposure in the study population

\section{Musculoskeletal disorders}

Alternative measures of vibration exposure

\section{Neck}

OR (95 \% CI)
Shoulder/upper arm

OR (95 \% CI)
Elbow/forearm Wrist/hand

OR (95 \% CI) 


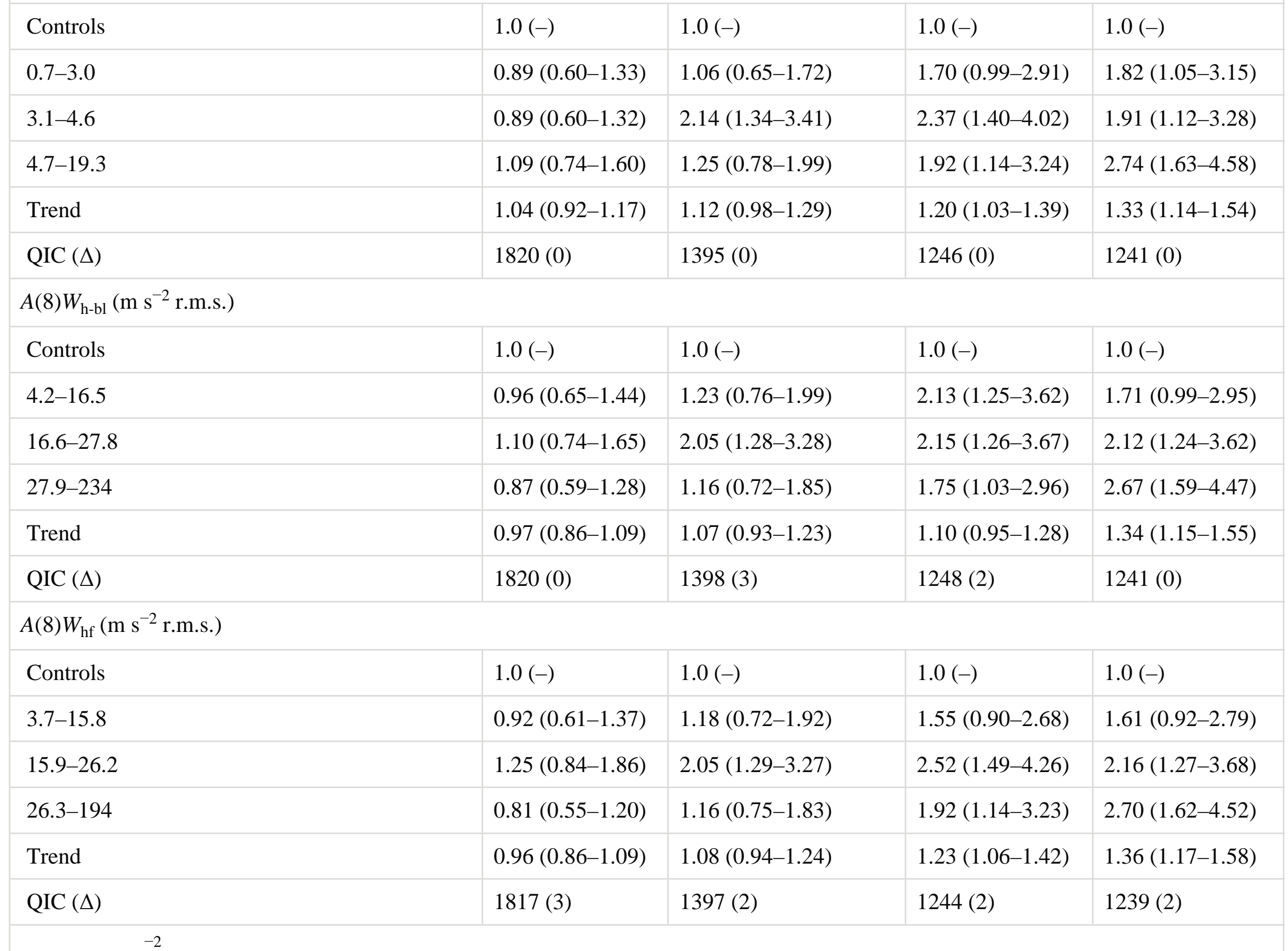


$A(8) W_{\mathrm{hT}}(\mathrm{m} \mathrm{s} \quad$ r.m.s.)

\begin{tabular}{|l|l|l|l|l|}
\hline Controls & $1.0(-)$ & $1.0(-)$ & $1.0(-)$ & $1.0(-)$ \\
\hline $3.4-14.3$ & $0.94(0.63-1.40)$ & $1.20(0.74-1.95)$ & $1.63(0.95-2.80)$ & $1.68(0.97-2.91)$ \\
\hline $14.4-23.9$ & $1.21(0.81-1.80)$ & $2.02(1.26-3.22)$ & $2.57(1.52-4.35)$ & $1.99(1.17-3.41)$ \\
\hline $24.0-180$ & $0.82(0.56-1.21)$ & $1.19(0.74-1.90)$ & $1.86(1.10-3.13)$ & $2.79(1.67-4.67)$ \\
\hline Trend & $0.96(0.85-1.08)$ & $1.08(0.94-1.24)$ & $1.20(1.04-1.40)$ & $1.36(1.17-1.58)$ \\
\hline QIC $(\Delta)$ & $1817(3)$ & $1398(3)$ & $1244(2)$ & $1239(2)$ \\
\hline
\end{tabular}

Odds ratios (OR) and robust $95 \%$ confidence intervals (95\% CI) were estimated by means of the generalised estimating equations method for repeated measures over time. See text for the definition of $A(8) W_{\mathrm{h}}, A(8) W_{\mathrm{h}-\mathrm{bl}}, A(8) W_{\mathrm{hf}}$, and $A(8) W_{\mathrm{hT}}$

OR adjusted by age at entry, body mass index, smoking and drinking habits, physical work load, psychological strain, neck/upper limb traumatic injuries or surgery, and follow-up time

QIC is the quasi-likelihood information criterion for the comparison between models. QIC difference ( $\Delta$ ) is calculated as the difference between the QIC value for a specific exposure model and the $A(8) W_{\mathrm{h}}$ model

In the vibration-exposed workers, $A(8) W_{\text {hi }}$ are expressed as tertile-based design variables

A trend for an increased risk of MSDs with the increase in the measures of vibration exposure was more evident for the wrist/hand (ORs 1.34-1.38) than for the elbow/forearm (ORs 1.10-1.23).

The QIC statistic did not reveal any substantial difference between the alternative measures of daily vibration exposure $\left(A(8) W_{\mathrm{h}}\right.$, $\left.A(8) W_{\mathrm{h}-\mathrm{bl}}, A(8) W_{\mathrm{hf}}, A(8) W_{\mathrm{hT}}\right)$ in the fit to the occurrence of neck and upper limb MSDs in the study population ( $\left.\Delta \mathrm{QIC} 0-3\right)$.

In addition to vibration exposure, multivariable data analysis showed that age, physical work load, and psychological strain, but not body mass index, smoking, or drinking habits, were significantly associated with neck and upper limb MSDs (results not shown). As an example, Table 4 shows that in all $A(8) W_{\text {hi }}$ models, age, hard physical work load, and high psychological strain were significant predictors of the occurrence of wrist/hand MSDs. No significant interactions between covariates were observed when appropriate product terms were included in the GEE logistic models. 


\section{Table 4}

Association between wrist/hand musculoskeletal disorders and individual- and work-related risk factors in the study population by alternative models of vibration exposure $\left(A(8) W_{\mathrm{hi}}\right)$

\begin{tabular}{|c|c|c|c|c|}
\hline Predictors & Model $A(8) W_{h}$ & Model $A(8) W_{\text {h-bl }}$ & Model $A(8) W_{\text {hf }}$ & Model $A(8) W_{h T}$ \\
\hline Body mass index ( $\left.>25 \mathrm{~kg} \mathrm{~m}^{-2}\right)$ & $0.70(0.47-1.07)$ & $0.70(0.46-1.07)$ & $0.71(0.47-1.08)$ & $0.71(0.47-1.08)$ \\
\hline Smoking (yes vs. no) & $1.21(0.89-1.66)$ & $1.19(0.86-1.62)$ & $1.16(0.85-1.60)$ & $1.16(0.85-1.60)$ \\
\hline \multicolumn{5}{|l|}{ Physical work load } \\
\hline No/mild (score 0-12) & $1.0(-)$ & $1.0(-)$ & $1.0(-)$ & $1.0(-)$ \\
\hline Moderate (score 13-25) & $1.42(0.83-2.44)$ & $1.41(0.82-2.42)$ & $1.42(0.83-2.44)$ & $1.42(0.83-2.44)$ \\
\hline \multicolumn{5}{|c|}{ Psychological strain in the last 7 days } \\
\hline No/mild (score 0-2) & $1.0(-)$ & $1.0(-)$ & $1.0(-)$ & $1.0(-)$ \\
\hline Moderate (score 3-5) & $1.37(0.89-2.12)$ & $1.38(0.90-2.13)$ & $1.39(0.90-2.14)$ & $1.39(0.90-2.14)$ \\
\hline High (score 6-8) & $2.13(1.09-4.16)$ & $2.11(1.07-4.16)$ & $2.02(1.02-4.01)$ & $2.02(1.02-4.01)$ \\
\hline Follow-up time (year) & $1.13(0.97-1.31)$ & $1.13(0.98-1.31)$ & $1.13(0.97-1.31)$ & $1.13(0.97-1.31)$ \\
\hline \multicolumn{5}{|c|}{$\begin{array}{l}\text { Odds ratios (OR) and robust } 95 \% \text { confidence intervals (95\% CI) were estimated by means of the generalised estimating equation } \\
\text { method for repeated measures over time }\end{array}$} \\
\hline
\end{tabular}


It is worth noting that unlike vibration exposure, high psychological strain was the strongest determinant of the occurrence of neck disorders in the study population (OR 6.36, $95 \%$ CI 2.90-13.9, according to the ISO model $\left(A(8) W_{\mathrm{h}}\right)$ ), (results not shown).

\section{Discussion}

This prospective cohort study showed an increased risk of upper limb MSDs in HTV workers compared with controls unexposed to vibration. Moreover, there was evidence for an increased occurrence of MSDs with the increase in alternative measures of vibration exposure.

\section{Vibration exposure and occupational MSDs}

An increased occurrence of MSDs associated with the use of vibratory tools has been observed in epidemiological studies of either working populations (Palmer et al. 2001) or worker groups occupationally exposed to HTV such as miners, shipyard and foundry workers, forestry operators, or stone workers (CEN 1996). In these studies, the prevalence of MSDs varied from 20 to $36 \%$ for the neck, 19-39\% for the shoulder/upper arm, 9-43\% for the elbow/forearm, and 17-36 \% for the wrist/hand. In accord with other epidemiological investigations, in this study increased risk estimates for MSDs in the HTV workers, compared to unexposed controls, were found mainly for the elbow/forearm and the wrist/hand (Bovenzi et al. 1991; Palmer et al. 2001).

Consistently with the results of previous reviews of epidemiological surveys, we did not find any significant association between neck disorders and exposure to HTV (Palmer and Smedley 2007; Mayer et al. 2012). Conversely, in this study, psychological strain in the previous 7 days was the most significant predictor of neck disorders over time, supporting the findings of other cohort and case-control studies that psychological distress and psychosomatic problems are major determinants of neck pain/symptoms in the general or working populations (Leclerc et al. 1999; van den Heuvel et al. 2005).

Studies of the association between shoulder disorders and exposure to HTV have produced contradictory results: some studies concluded for no or insufficient evidence for such an association, while others reported an increased occurrence of shoulder troubles in HTV workers compared with unexposed controls (Mayer et al. 2012; Van Rijn et al. 2010). In the present study, the association between shoulder disorders and HTV exposure was weaker than those for the elbow/forearm and the wrist/hand. 
It is prevalent opinion among researchers that occupational MSDs in the neck and upper limbs are of multifactorial origin (Widanarko et al. 2014). Demographic characteristics, biomechanical, physical, psychological/psychosocial, and organisational risk factors at the workplace play a variable role in the etiopathogenesis of neck and upper limb MSDs according to differences in individual susceptibility, job title, industrial trade, and socio-economic and cultural environments. The findings of this study tend to support the multifactorial aspects involved in the development of occupational MSDs in HTV workers. In addition to vibration, age, excessive physical work load, and high psychological strain were significant predictors of the occurrence of MSDs over the study period. Moreover, data analysis showed no significant interactions between these risk factors, suggesting that they may contribute independently of each other to the onset of MSDs, although to a different extent in the distal versus proximal anatomical sites of the upper limbs.

\section{Alternative frequency weightings of hand-transmitted vibration and occupational MSDs}

In this prospective cohort study, all alternative measures of daily vibration exposure calculated by means of four different frequency weightings of HTV were significant predictors of the occurrence of MSDs in the distal anatomical sites of the upper limbs, with a better trend of increasing risk with the increase in the alternative $A(8) W_{\text {hi }}$ for the wrist/hand than for the elbow/forearm. Nevertheless, a measure of statistical fit showed no remarkable difference in the performance of the four alternative frequency weightings of HTV for the prediction of the occurrence of neck and upper limb MSDs over the follow-up period.

This finding may be explained, at least partially, considering the biodynamic and physiological responses of the hand-arm system to vibration exposure. The greater excess risk for the distal structures rather than for the proximal sites of the upper limbs may be ascribed, at least partially, to the biodynamic finding that the transmissibility of vibration along the human hand-arm system decreases with the distance from the source, and the transmission of mechanical energy to the upper arm and shoulder is strongly attenuated compared with the vibration transmission to the elbow/forearm and the wrist/hand, mainly for vibration frequencies higher than $50 \mathrm{~Hz}$ (Sörensson and Burström 1997; Welcome et al. 2015).

Transmissibility measurements have shown that the hand and wrist operate as a low-pass filter since low-frequency vibration $(<40$ $50 \mathrm{~Hz}$ ) is effectively transmitted to the upper limbs with a progressive attenuation from the wrist to the shoulder, while most of the vibrational energy in the mid- and high-frequency range $(>50-100 \mathrm{~Hz})$ is either stored or dissipated in the carpal, metacarpal, and phalangeal areas (Concettoni and Griffin 2009; Welcome et al. 2015). It has been shown that vibration stress induces large 
relative motions and deformations of adjacent soft tissues at the major biodynamic resonances in the fingers (100-200 Hz), the hand dorsum/palm and wrist (20-40 Hz), the forearm and elbow (16-30 Hz), and the upper arm and shoulder (8-12 Hz), depending on the vibration direction, the measurement points on the skin surface of the upper limbs, the hand and arm postures, and the applied forces (grip, push, and contact forces), (Sörensson and Burström 1997; Dong et al. 2005; Concettoni and Griffin 2009; Welcome et al. 2015). On the basis of the biodynamic responses of the structures of the upper limbs to vibration, it has been suggested that the ISO frequency weighting of HTV $\left(a_{\mathrm{h}}\left(W_{\mathrm{h}}\right)\right)$, which gives relatively more weight to low-frequency vibration $(6.3-$ $20 \mathrm{~Hz}$ ) than to intermediate- and high-frequency vibration (25-1250 Hz), seems appropriate to assess the risk of wrist and elbow disorders in HTV workers (Dong et al. 2005). In this context, a German guideline for exposure to hand-arm vibration (VDI 2057-2 2002) suggests to measure the ISO frequency-weighted acceleration of vibration in the frequency range 8-50 Hz (when the proportion of $a_{\mathrm{h}}\left(W_{\mathrm{h}}\right)_{8-50 \mathrm{~Hz}}$ is more than $75 \%$ of the total $\left.a_{\mathrm{h}}\left(W_{\mathrm{h}}\right)_{8-1000 \mathrm{~Hz}}\right)$ to establish whether there is a risk of osteoarticular diseases in the wrist and elbow joints of the HTV workers. It should be noted, however, that the term "musculoskeletal disorders", as defined in this and other similar studies, is not exactly equivalent to that of "osteoarticular diseases" as denoted in either the above-mentioned VDI guideline (2002) or the European schedule of occupational diseases (2003); rather, the term "osteoarticular disease" refers to radiological findings of degenerative changes in the bones and joints of the elbow (osteoarthrosis, exostosis at the sites of tendon insertions) and the wrist/hand (osteoarthrosis, enostosis, vacuoles) observed in some groups of HTV workers (Gemne and Saraste 1987), mainly in those who operated percussive tools which generate high acceleration magnitudes in the low-frequency range $(<40-50 \mathrm{~Hz})$. Nonetheless, there is experimental evidence that higher vibration frequencies $(>100 \mathrm{~Hz})$ can also induce pathological changes in the bone tissue. In a rat-tail model with the rat caudal vertebrae vibrated at 125 and $250 \mathrm{~Hz}$ with an acceleration magnitude of $49 \mathrm{~m} \mathrm{~s}^{-2}$ r.m.s. for 1, 5, or 20 days, structural damage (changes in osteocyte and empty lacunae counts) and biochemical alterations (nitrotyrosine-mediated oxidative injury) in the cortical bone were found to increase with the increase in the frequency and the duration of vibration (Peelukhana et al. 2015).

Physiological investigations have revealed that vibration stimuli applied at a muscle belly or a tendon of the upper limbs can elicit a reflex muscular contraction, known as the "tonic vibration reflex" (TVR). This response results from the excitation of the primary endings (Ia fibres) in the muscle spindle and is mediated through complex spinal and supra-spinal reflex arc mechanisms involving monosynaptic and polysynaptic pathways (Homma et al. 1971). It has been shown that the TVR in the finger and wrist flexor muscles can be activated by vibration frequencies in the range 40-200 Hz; the tonic contraction activity increases with the vibration frequency up to 100-150 Hz and thereafter declines (Park and Martin 1993). Spectral analysis of electromyographic (EMG) recordings has shown a harmonic synchronisation (1:1) between the vibration frequency and the firing rate of the motor units for frequencies $\leq 100 \mathrm{~Hz}$, while subharmonic peaks of the EMG signals are predominant at higher frequencies of the vibration 
stimulus (200 Hz), (Martin and Park 1997). Previous laboratory studies have also shown an increase in the electrical activity of the muscles of the hand, forearm, and upper arm during exposure to wide-band random vibration signals alone or in combination with different degrees of push and grip forces (Hartung et al. 1993). On the basis of these findings, it has been hypothesised that occupational exposure to harmful HTV, combined with a tight grip force and/or repetitive movements when operating vibratory tools, can cause muscle and tendon stress and fatigue increasing the risk of soft-tissue disorders in the upper limbs of the exposed workers (Park and Martin 1993). A biological plausibility to this hypothesis is provided by a murine experimental model in which exposure to a vibration of $80 \mathrm{~Hz}$ with a magnitude of $32 \mathrm{~m} \mathrm{~s}^{-2}$ r.m.s. (unweighted acceleration), delivered for $5 \mathrm{~h}$ daily during five consecutive days, was found to induce severe injury and degeneration, necrosis included, in the muscles fibres of the vibrated plantar muscles of the experimental animals (Necking et al. 1992).

Overall, the findings of biodynamic and electrophysiological studies seem to suggest that the occurrence of MSDs in HTV workers is associated with (1) the transmission and absorption of vibration energy in the soft tissues of the upper limbs, which are in turn influenced by the hand-arm posture and the magnitude of the push and grip forces exerted on the tool handle; (2) the reaction of muscles and tendons to vibration stimuli, resulting in localised stress and fatigue.

In the present study, all four alternative frequency weightings of HTV were good predictors of the occurrence over time of MSDs in the distal sites of the upper limbs of the exposed workers. Since the resonances in the soft tissues of the hand (dorsum/palm), wrist, and elbow occur in the frequency range 16-40 Hz, and the peak of the TVR response is at 100-150 Hz, it is not surprising that the four alternative frequency weightings provided a similar fitting to epidemiological data because they give alternatively more weight to either low-frequency vibration $\left(a_{\mathrm{h}}\left(W_{\mathrm{h}}\right)\right)$ or intermediate- and high-frequency vibration $\left(a_{\mathrm{h}}\left(W_{\mathrm{h}-\mathrm{bl}}\right), a_{\mathrm{h}}\left(W_{\mathrm{hf}}\right), a_{\mathrm{h}}\left(W_{\mathrm{hT}}\right)\right)$, i.e. within the range of vibration frequencies $(8-200 \mathrm{~Hz})$ which are experimentally associated with an increased strain in the soft tissues of the upper limbs.

As aforementioned, it is to point out that HTV is one component of the multifactorial origin of MSDs in the exposed workers; as observed in this and other studies, additional physical work loads (e.g. force, repetitiveness, and posture) and psychological/psychosocial risk factors are also important contributors to the etiopathogenesis of MSDs in HTV workers (van den Heuvel et al. 2005; Widanarko et al. 2014).

\section{Uncertainties and limitations of the study}

In this study, we focused on the use of different frequency weightings of vibration to estimate the risk of musculoskeletal injuries in 
the upper limbs of HTV workers. We recognise that in addition to frequency, vibration magnitude, direction, and duration are also important variables to predict the human response to vibration. The separate effects of these variables on the human sensitivity to vibration can be primarily studied in the laboratory since it is unfeasible to explore the influence of each variable in a working environment. Moreover, in this study, the severity of daily exposure to HTV $[A(8)]$ has been evaluated according to the methods suggested in the current ISO standard (ISO 5349-1 2001) which assumes an equal sensitivity to the three directions of vibration $(x-, y$-, and $z$-axes) and a second-power time dependency over the work day (i.e. r.m.s. averaging). Considerations on the accuracy of these assumptions are beyond the scope of this paper; here, they are mentioned because the current frequency, direction, and duration weighting methods for predicting the adverse effects of human vibration are still controversial (Griffin 1997).

Potential sources of bias in this study have been reported in previous papers of the VIBRISKS cohort (Bovenzi 2010; Bovenzi et al. 2011). In our study, work-related physical loading other than mechanical vibration was evaluated by subjective judgement of the frequency of adverse movements and awkward postures. Since the association between MSDs and physical load risk factors was evaluated mainly on the basis of self-reported working postures and manual material handling, potential bias for spurious associations between occupational exposures and MSD outcomes cannot be ruled out. Although the role of the questionnaire as an instrument for assessing occupational physical stressors is a debated issue (Burdorf and van der Beek 1999), nevertheless questionnaire methods may offer a means for the evaluation of cumulative exposure over time, a variable which cannot be estimated by direct observations or measurements.

In this study, vibration measurements were taken on the tools currently used by the forestry and stone workers, and this may be a source of uncertainty. However, the vibration magnitudes measured in the tools of the present study are broadly comparable with those reported in recent and past investigations and in vibration guidelines (Griffin et al. 2006).

Quantification of duration of exposure to HTV may be difficult because recall bias cannot be ruled out when daily exposure duration is estimated by means of questionnaire or direct interview of employees and employers. Several investigations have reported that HTV workers tend to overestimate their actual daily duration of exposure to the vibration from tools (Palmer et al. 2000). To reduce, at least partially, this bias, a survey was conducted in the field over an entire week period by supervisors who used a stopwatch method recommended by the EU guide on HTV to measure the time the hands of the worker are actually in contact with the operating tool (Griffin et al. 2006). Partial vibration exposures were determined from the magnitude and duration of representative operations performed with specific tools, and then, the overall daily vibration exposure was calculated according to the EU guidance (Griffin et al. 2006). 
Other sources of uncertainties, such as the short duration of the follow-up time (3 years), feedback bias in longitudinal studies (i.e. modification of exposure in symptomatic workers), or the assumption about missing at random data, have been discussed elsewhere (Bovenzi 2010).

\section{Conclusions}

In this study, there was evidence for significant exposure-response relationships between HTV exposure and the occurrence of MSDs in the elbow/forearm and the wrist/hand. A measure of model selection did not reveal any substantial difference in the prediction of neck and upper limb MSDs between measures of daily vibration exposure calculated with four alternative frequency weightings of acceleration magnitude.

\section{Acknowledgments}

This research was supported by the European Commission under the Quality of Life and Management of Living Resources programme-Project No. QLK4-2002-02650 (VIBRISKS)

Compliance with ethical standards

Conflict of interest The authors declare no competing interests.

\section{References}

Bovenzi M (2010) A prospective cohort study of exposure-response relationship for vibration-induced white finger. Occup Environ Med 67:38-46

Bovenzi M, Zadini A, Franzinelli A, Borgioni F (1991) Occupational musculoskeletal disorders in the neck and upper limbs of forestry workers exposed to hand-arm vibration. Ergonomics 34:547-562

Bovenzi M, Pinto I, Picciolo F, Mauro M, Ronchese F (2011) Frequency weightings of hand-transmitted vibration for predicting vibration-induced white finger. Scand J Work Environ Health 37:244-252 
Burdorf A, van der Beek AJ (1999) In musculoskeletal epidemiology are we asking the unanswerable in questionnaires on physical load? Scand J Work Environ Health 25:81-83

Burnham KP, Anderson DR (2004) Multimodel inference: understanding AIC and BIC in model selection. Sociol Methods Res 33:261-304

Comité Européen de Normalisation (CEN) (1996) Mechanical vibration—guide to the health effects of vibration on the human body. CR Report 12349. CEN, Brussels

Commission Recommendation of 19 September 2003 concerning the European schedule of occupational diseases (2003/670/EC). Official Journal of the European Union, L 238/28, 25.9.2003

Concettoni E, Griffin MJ (2009) The apparent mass and mechanical impedance of the hand and the transmission of vibration to the fingers, hand, and arm. J Sound Vib 325:664-678

Cui J (2007) QIC program and model selection in GEE analyses. Stata J 7:209-220

Directive 2002/44/EC of the European Parliament and the Council of 25 June 2002 on the minimum health and safety requirements regarding the exposure of workers to the risks arising from physical agents (vibration) (16th individual Directive within the meaning of Article 16(1) of Directive 89/391/EEC). Official Journal of the European Communities, L 117/13, 6.7.2002

Dong RG, Welcome DE, Wu JZ (2005) Frequency weightings based on biodynamics of fingers-hand-arm system. Ind Health 43:516-526

Gemne G, Saraste H (1987) Bone and joint pathology in workers using hand-held vibrating tools. An overview. Scand J Work Environ Health 13:290-300

Griffin MJ (1997) Measurement, evaluation, and assessment of occupational exposures to hand-transmitted vibration. Occup Environ Med 54:73-89 
Griffin MJ, Howarth HVC, Pitts PM, Fischer S, Kaulbars U, Donati P, Bereton PF (2006) Guide to good practice on hand-arm vibration. European Commission Directorate General Employment, Social Affairs and Equal Opportunities (contract

VC/2004/0341)

Harrington JM, Carter JT, Birrell L, Gompertz D (1998) Surveillance case definitions for work-related upper limb pain syndromes. Occup Environ Med 55:264-271

Hartung E, Dupuis H, Scheffer M (1993) Effects of grip and push forces on the acute response on the hand-arm system under vibrating conditions. Int Arch Occup Environ Health 64:463-467

Homma S, Kanda K, Watanabe S (1971) Tonic vibration reflex in human and monkey subjects. Jpn J Physiol 21:419-430

Huisstede BMA, Miedema HS, Verhagen AP, Koes BW, Verhaar JAN (2007) Multidisciplinary consensus on the terminology and classification of complaints of the arm, neck and/or shoulder. Occup Environ Med 64:313-319

International Organization for Standardization (ISO) (2001) Mechanical vibration-measurement and evaluation of human exposure to hand-transmitted vibration—-part 1: general requirements. ISO 5349-1. ISO, Geneva

Leclerc A, Niedhammer I, Landre M-F, Ozguler A, Etore P, Pietri-Taleb F (1999) One-year predictive factors for various aspects of neck disorders. Spine 24:1455-1462

Martin BJ, Park H-S (1997) Analysis of the tonic vibration reflex: influence of vibration variables on motor unit synchronization and fatigue. Eur J Appl Physiol 75:504-511

Mayer J, Kraus T, Ochsmann E (2012) Longitudinal evidence for the association between work-related physical exposures and neck and/or shoulder complaints: a systematic review. Int Arch Occup Environ Health 85:587-603

Necking LE, Dahlin LB, Fridén J, Lundborg G, Lundström R, Thornell LE (1992) Vibration-induced muscle injury. J- Hand Surg 17B:270-274 
Palmer KT, Smedley J (2007) Work relatedness of chronic neck shoulder pain with physical findings—a systematic review. Scand J Work Environ Health 33:165-191

Palmer KT, Haward B, Griffin MJ, Bednall H, Coggon D (2000) Validity of self reported occupational exposure to hand transmitted and whole body vibration. Occup Environ Med 57:237-241

Palmer KT, Griffin MJ, Syddall HE, Pannett B, Cooper C, Coggon D (2001) Exposure to hand-transmitted vibration and pain in the neck and upper limbs. Occup Med 51:464-467

Park H-S, Martin BJ (1993) Contribution of the tonic vibration reflex to muscle stress and muscle fatigue. Scand J Work Environ Health 19:35-42

Peelukhana SV, Goenka S, Kim B, Kim J, Bhattacharya A, Stringer KF, Banerjee RK (2015) Effect of higher frequency components and duration of vibration on bone tissue alterations in the rat-tail model. Ind Health 53:245-259

Pitts P, Mason HJ, Poole KA, Young CE (2012) Relative performance of frequency weighting $W_{\mathrm{h}}$ and candidates for alternative frequency weightings for predicting the occurrence of hand-transmitted vibration induced injuries. Ind Health 50:388-396

Punnett L, Gold J, Katz JN, Gore R, Wegman DH (2004) Ergonomic stressors and upper extremity musculoskeletal disorders in automobile manufacturing: a one year follow up study. Occup Environ Med 61:668-674

Sörensson A, Burström L (1997) Transmission of vibration energy to different parts of the human hand-arm system. Int Arch Occup Environ Health 70:199-204

Tominaga Y (2005) New frequency weighting of hand-arm vibration. Ind Health 43:509-515

van den Heuvel SG, van der Beek AJ, Blatter BM, Hoogendoorn WE, Bongers PM (2005) Psychosocial work characteristics in relation to neck and upper limb symptoms. Pain 114:47-53

Van Rijn RM, Huisstede BMA, Koes BW, Burdorf A (2010) Associations between work-related factors and specific disorders 
Verein Deutscher Ingenieure (VDI) (2002) Human exposure to mechanical vibration—part 2: hand-arm vibration. Annex E. VDI 2057. VDI, Düsseldorf

VIBRISKS (2007) Risks of Occupational Vibration Exposures. FP5 Project No. QLK4-2002-02650. European Commission Quality of Life and Management of Living Resources Programme. Southampton, UK: Human Factors Research Unit, Institute of Sound and Vibration Research, University of Southampton [updated 28 February 2007; cited 1 April 2014].

http://www.vibrisks.soton.ac.uk

Welcome DE, Dong RG, Xu XS, Warren C, McDowell TW, Wu JZ (2015) An examination of the vibration transmissibility of the hand-arm system in three orthogonal directions. Int J Ind Ergon 45:21-34

Widanarko B, Legg S, Devereux J, Stevenson M (2014) The combined effect of physical, psychosocial/organisational and/or environmental risk factors on the presence of work-related musculoskeletal symptoms and its consequences. Appl Ergon 45:1610-1621 